Research Article

\title{
Four-Dimensional Semi-Riemannian Szabó Manifolds
}

\author{
Abdoul Salam Diallo $\mathbb{D}^{1}$ and Punam Gupta $\mathbb{D}^{2}$ \\ ${ }^{1}$ Université Alioune Diop de Bambey, UFR SATIC, Département de Mathématiques, \\ Equipe de Recherche en Analyse Non Linéaire et Géométrie, B. P. 30, Bambey, Senegal \\ ${ }^{2}$ Department of Mathematics \& Statistics, School of Mathematical \& Physical Sciences, Dr. Harisingh Gour University, \\ Sagar 470003, Madhya Prades, India
}

Correspondence should be addressed to Abdoul Salam Diallo; abdoulsalam.diallo@uadb.edu.sn

Received 20 October 2020; Accepted 14 December 2020; Published 31 December 2020

Academic Editor: Ljubisa Kocinac

Copyright (c) 2020 Abdoul Salam Diallo and Punam Gupta. This is an open access article distributed under the Creative Commons Attribution License, which permits unrestricted use, distribution, and reproduction in any medium, provided the original work is properly cited.

In this paper, we prove that the deformed Riemannian extension of any affine Szabó manifold is a Szabó pseudo-Riemannian metric and vice versa. We prove that the Ricci tensor of an affine surface is skew-symmetric and nonzero everywhere if and only if the affine surface is Szabó. We also find the necessary and sufficient condition for the affine Szabó surface to be recurrent. We prove that, for an affine Szabó recurrent surface, the recurrence covector of a recurrence tensor is not locally a gradient.

\section{Introduction}

Let $T^{*} M$ be the cotangent bundle of $n$-dimensional manifold $M$ with a torsion-free affine connection $\nabla$. Patterson and Walker [1] introduced the notion of Riemannian extensions and showed how to construct a pseudo-Riemannian metric on the $2 n$-dimensional cotangent bundle of any $n$-dimensional manifold with a torsion-free connection. Afifi [2] studied the local properties of Riemannian extension of connected affine spaces. Riemannian extensions were also studied by García-Río et al. [3] for Osserman manifolds. Diallo [4] found the fruitful results for the Riemannian extension of an affine Osserman connection on 3-dimensional manifolds. In [5], the authors generalized the Riemannian extension to the deformed Riemannian extensions. In the recent paper [6], we construct example of pseudoRiemannian Szabó metrics of signature $(2,2)$ by using the deformed Riemannian extension, whose Szabó operators are nilpotent. The Riemannian extension can be constructed with the help of the coefficients of the torsion-free affine connection. For Riemannian extensions, also see [7-9]. For deformed Riemannian extensions, also see [10-12].

In this paper, we study the deformed Riemannian extensions of affine Szabó manifold. Our paper is organized as follows. In Section 2, we recall some basic definitions and results on the deformed Riemannian extension. In Section 3, we provide some known results on affine Szabó manifolds. We prove that the Ricci tensor of an affine surface is skewsymmetric and nonzero everywhere if and only if the affine surface is Szabó. We also find the necessary and sufficient condition for the affine Szabó surface to be recurrent. We prove that for an affine Szabó recurrent surface, the recurrence covector of a recurrence tensor is not locally a gradient. Finally, in Section 4, we prove that the deformed Riemannian extension of any affine Szabó manifold is a Szabó pseudo-Riemannian metric and vice versa.

Throughout this paper, all manifolds, tensors fields, and connections are always assumed to be $\mathrm{E}^{\infty}$-differentiable.

\section{Deformed Riemannian Extensions}

Let $T^{*} M$ be the cotangent bundle of $n$-dimensional affine manifold $M$ with torsion-free affine connection $\nabla$ and let $\pi: T^{*} M \longrightarrow M$ be the natural projection defined by

$$
\pi(p, \omega)=p \in M \text { and }(p, \omega) \in T^{*} M \text {. }
$$

A system of local coordinates $\left(U, u_{i}\right), \quad i=1, \ldots, n$ around $p \in M$ induces a system of local coordinates $\left(\pi^{-1}(U), u_{i}, u_{i^{\prime}}=\omega_{i}\right), \quad i^{\prime}=n+i=n+1, \ldots, 2 n, \quad$ around 
$(p, \omega) \in T^{*} M$, where $\omega_{i}$ are components of covectors $\omega$ in each cotangent space $T_{p}^{*} M, p \in U$, with respect to the natural coframe $\left\{d u^{i}\right\}$. Let $\partial_{i}=\partial / \partial u_{i}$ and $\partial_{i^{\prime}}=\partial / \partial \omega_{i}, \quad i=i, \ldots, n$, then, at each point $(p, \omega) \in T^{*} M$,

$$
\left\{\left(\partial_{1}\right)_{(p, \omega)}, \ldots,\left(\partial_{n}\right)_{(p, \omega)},\left(\partial_{1^{\prime}}\right)_{(p, \omega)}, \ldots,\left(\partial_{n^{\prime}}\right)_{(p, \omega)}\right\},
$$

is a basis for the cotangent space $\left(T^{*} M\right)_{(p, \omega)}$. For more details on the geometry of cotangent bundle, see [13].

The Riemannian extension $g_{\nabla}$ is the pseudo-Riemannian metric on $T^{*} M$ of neutral signature $(n, n)$ characterized by the identity [5].

$$
g_{\nabla}\left(X^{C}, Y^{C}\right)=-\iota\left(\nabla_{X} Y+\nabla_{Y} X\right)
$$

where $X^{C}$ is a complete lift of the vector field $X$ on $M$, and the function $\iota X: T^{*} M \longrightarrow \mathbb{R}$ is defined by

$$
\iota X(p, \omega)=\omega\left(X_{p}\right)
$$

For more details, see [5]. In the locally induced coordinates $\left(u_{i}, u_{i^{\prime}}\right)$ on $\pi^{-1}(U) \subset T^{*} M$, the Riemannian extension [1] is expressed by

$$
g_{\nabla}=\left(\begin{array}{rr}
-2 u_{k^{\prime}} \Gamma_{i j}^{k} & \delta_{i}^{j} \\
\delta_{i}^{j} & 0
\end{array}\right),
$$

with respect to the basis $\left\{\partial_{1}, \ldots, \partial_{n}, \partial_{1^{\prime}}, \ldots, \partial_{n^{\prime}}\right\}\left(i, j, k=1, \ldots, n ; k^{\prime}=k+n\right)$, where $\Gamma_{i j}^{k}$ are the coefficients of the torsion-free affine connection $\nabla$ with respect to $\left(U, u_{i}\right)$ on $M$.

Riemannian extensions provide a link between affine and pseudo-Riemannian geometries. Therefore, by using the properties of the Riemannian extension $g_{\nabla}$, we investigate the properties of the affine connection $\nabla$. Similarly, $(M, \nabla)$ is locally symmetric if and only if $\left(T^{*} M, g_{\nabla}\right)$ is locally symmetric. In the same way, $(M, \nabla)$ is projectively flat if and only if $\left(T^{*} M, g_{\nabla}\right)$ is locally conformally flat [14].

Let $\phi$ be a symmetric $(0,2)$-tensor field on an affine manifold $(M, \nabla)$. In [14], the authors introduced a deformation of the Riemannian extension by means of a symmetric $(0,2)$-tensor field $\phi$ on $M$. They considered the cotangent bundle $T^{*} M$ equipped with the metric $g_{\nabla}+\pi^{*} \phi$, which is called the deformed Riemannian extension.

The deformed Riemannian extension denoted $g_{(\nabla, \phi)}$ which is the metric of neutral signature $(n, n)$ on the cotangent bundle given by

$$
g_{(\nabla, \phi)}=g_{\nabla}+\pi^{*} \phi .
$$

In local coordinates, the deformed Riemannian extension is given by

$$
g_{(\nabla, \phi)}=\left(\begin{array}{cc}
\phi_{i j}(u)-2 u_{k^{\prime}} \Gamma_{i j}^{k} & \delta_{i}^{j} \\
\delta_{i}^{j} & 0
\end{array}\right),
$$

with respect to the basis $\left\{\partial_{1}, \ldots, \partial_{n}, \partial_{1^{\prime}}, \ldots, \partial_{n^{\prime}}\right\}$, $\left(i, j, k=1, \ldots, n ; k^{\prime}=k+n\right)$, where $\Gamma_{i j}^{k}$ are the coefficients of the torsion-free affine connection and $\nabla$ and $\phi_{i j}$ are the local components of the symmetric $(0,2)$-tensor field $\phi$. Equivalently,

$$
\begin{aligned}
& g_{(\nabla, \phi)}\left(\partial_{i}, \partial_{j}\right)=\phi_{i j}(u)-2 u_{k^{\prime}} \Gamma_{i j}^{k}, \\
& g_{(\nabla, \phi)}\left(\partial_{i}, \partial_{j^{\prime}}\right)=\delta_{i}^{j}, \\
& g_{(\nabla, \phi)}\left(\partial_{i^{\prime}}, \partial_{j^{\prime}}\right)=0 .
\end{aligned}
$$

Note that the crucial terms $g_{(\nabla, \phi)}\left(\partial_{i}, \partial_{j}\right)$ now no longer vanish on the 0 -section, which was the case for the Riemannian extension, and the Walker distribution is the kernel of the projection from $T^{*} M$ :

$$
D=\operatorname{ker}\left\{\pi^{*}\right\}=\operatorname{Span}\left\{\partial_{i^{\prime}}\right\} .
$$

In the deformed Riemannian extension, the tensor $\phi$ plays an important role. If the underlying connection is flat, the deformed Riemannian extension need not be flat [5]. Deformed Riemannian extensions have nilpotent Ricci operator; therefore, they are Einstein if and only if they are Ricci flat. So, deformed Riemannian extension can be used to construct nonflat Ricci pseudo-Riemannian manifolds [14].

\section{The Affine Szabó Manifolds}

Let $(M, \nabla)$ be an affine manifold and $X \in \Gamma\left(T_{p} M\right)$. The affine Szabó operator $S^{\nabla}(X)$ [15] with respect to $X$ is a function from $T_{p} M$ to $T_{p} M, p \in M$, defined by

$$
S^{\nabla}(X) Y=\left(\nabla_{X} \mathscr{R}^{\nabla}\right)(Y, X) X,
$$

for any vector field $Y$ and where $\mathscr{R}^{\nabla}$ is the curvature operator of the affine connection $\nabla$. The affine Szabó operator satisfies $S^{\nabla}(X) X=0$ and $S^{\nabla}(\beta X)=\beta^{3} S(X)$, for $\beta \in \mathbb{R}^{*}$. If $Y=\partial_{m}$, for $m=1,2, \ldots, n$, and $X=\sum_{i} \alpha_{i} \partial_{i}$, we have

$$
S^{\nabla}(X) \partial_{m}=\sum_{i, j, k=1}^{n} \alpha_{i} \alpha_{j} \alpha_{k}\left(\nabla_{i} \mathscr{R}^{\nabla}\right)\left(\partial_{m}, \partial_{j}\right) \partial_{k},
$$

where $\nabla_{i}=\nabla_{\partial_{i}}$.

Let $(M, \nabla)$ be an affine manifold and $p \in M .(M, \nabla)$ is said to affine Szabó at $p \in M$ if the affine Szabó operator $S^{\nabla}$ has the same characteristic polynomial for every vector field $X$ on $M$. If $(M, \nabla)$ is affine Szabó at each $p \in M$, then $(M, \nabla)$ is known as affine Szabó. For more details, see [16].

Now, we give a known result for later use.

Theorem 1 (see [17]). Let $(M, \nabla)$ be an $n$-dimensional affine manifold and $p \in M$. Then, $(M, \nabla)$ is affine Szabó at $p \in M$ if and only if the characteristic polynomial of the affine Szabó operator $S^{\nabla}$ is $P_{\lambda}\left[S^{\nabla}(X)\right]=\lambda^{n}$, for every $X \in T_{p} M$.

We have a complete description of affine Szabó surfaces.

Theorem 2 (see [17]). Let $\Sigma=(M, \nabla)$ be an affine surface. Then, $\Sigma$ is affine Szabo at $p \in M$ if and only if the Ricci tensor of $(M, \nabla)$ is cyclic parallel at $p \in M$.

Next, we investigate some particular case. The curvature of an affine surface is encoded by its Ricci tensor. We fix coordinates $\left(u_{1}, u_{2}\right)$ on $\Sigma$ and let $\nabla_{\partial_{i}} \partial_{j}=\Gamma_{i j}^{k} \partial_{k}$, for $i, j, k=1,2$, where $\Gamma_{i j}^{k}=\Gamma_{i j}^{k}\left(u_{1}, u_{2}\right)$. Then, a straightforward 
calculation shows that the components of the curvature tensor $\mathscr{R}$ are given by

$$
\mathscr{R}\left(\partial_{1}, \partial_{2}\right) \partial_{1}=\rho_{21} \partial_{1}-\rho_{11} \partial_{2} \text { and } \mathscr{R}\left(\partial_{1}, \partial_{2}\right) \partial_{2}=\rho_{22} \partial_{1}-\rho_{12} \partial_{2} \text {, }
$$

where $\rho_{i j}, i, j=1,2$ are the components of the Ricci tensor given

$$
\begin{aligned}
& \rho_{21}=\partial_{1} \Gamma_{12}^{1}-\partial_{2} \Gamma_{11}^{1}+\Gamma_{12}^{1} \Gamma_{12}^{2}-\Gamma_{11}^{2} \Gamma_{22}^{1}, \\
& \rho_{11}=-\left(\partial_{1} \Gamma_{12}^{2}-\partial_{2} \Gamma_{11}^{2}+\Gamma_{11}^{2} \Gamma_{12}^{1}+\Gamma_{12}^{2} \Gamma_{12}^{2}-\Gamma_{11}^{1} \Gamma_{12}^{2}-\Gamma_{11}^{2} \Gamma_{22}^{2}\right), \\
& \rho_{22}=\partial_{1} \Gamma_{22}^{1}-\partial_{2} \Gamma_{12}^{1}+\Gamma_{11}^{1} \Gamma_{22}^{1}+\Gamma_{12}^{1} \Gamma_{22}^{2}-\Gamma_{12}^{1} \Gamma_{12}^{1}-\Gamma_{12}^{2} \Gamma_{22}^{1}, \\
& \rho_{12}=-\left(\partial_{1} \Gamma_{22}^{2}-\partial_{2} \Gamma_{12}^{2}+\Gamma_{11}^{2} \Gamma_{22}^{1}-\Gamma_{12}^{1} \Gamma_{12}^{2}\right) .
\end{aligned}
$$

Let $X=\alpha_{1} \partial_{1}+\alpha_{2} \partial_{2}$ be a vector field on $\Sigma$. It is easy to check that the affine Szabó operator $S(X)$ expresses, with respect to the basis $\left\{\partial_{1}, \partial_{2}\right\}$, as

$$
\left(S^{\nabla}(X)\right)=\left(\begin{array}{ll}
A & B \\
C & D
\end{array}\right) .
$$

where the coefficients $A, B, C$, and $D$ are given by

$$
\begin{aligned}
A= & \alpha_{1}^{2} \alpha_{2}\left[\partial_{1} \rho_{21}-\left(\Gamma_{11}^{1}+\Gamma_{12}^{2}\right) \rho_{21}-\Gamma_{12}^{1} \rho_{11}-\Gamma_{11}^{2} \rho_{22}\right] \\
& +\alpha_{1} \alpha_{2}^{2}\left[\partial_{2} \rho_{21}+\partial_{1} \rho_{22}-\left(\Gamma_{12}^{1}+\Gamma_{22}^{2}\right) \rho_{21}\right. \\
& \left.-\left(\rho_{12}+\rho_{21}\right) \Gamma_{12}^{1}-\Gamma_{22}^{1} \rho_{11}-3 \Gamma_{12}^{2} \rho_{22}\right] \\
& +\alpha_{2}^{3}\left[\partial_{2} \rho_{22}-2 \Gamma_{22}^{2} \rho_{22}-\left(\rho_{12}+\rho_{21}\right) \Gamma_{22}^{1}\right] \\
B= & \alpha_{1}^{2} \alpha_{2}\left[-\partial_{1} \rho_{11}+2 \Gamma_{11}^{1} \rho_{11}+\left(\rho_{12}+\rho_{21}\right) \Gamma_{11}^{2}\right] \\
& +\alpha_{1} \alpha_{2}^{2}\left[-\partial_{2} \rho_{11}-\partial_{1} \rho_{12}+3 \Gamma_{12}^{1} \rho_{11}+\Gamma_{11}^{2} \rho_{22}\right. \\
& \left.+\left(\rho_{12}+\rho_{21}\right) \Gamma_{12}^{2}+\left(\Gamma_{11}^{1}+\Gamma_{12}^{2}\right) \rho_{12}\right] \\
& +\alpha_{2}^{3}\left[-\partial_{2} \rho_{12}+\Gamma_{22}^{1} \rho_{11}+\Gamma_{12}^{2} \rho_{22}+\left(\Gamma_{12}^{1}+\Gamma_{22}^{2}\right) \rho_{12}\right] \\
C= & \alpha_{1}^{3}\left[-\partial_{1} \rho_{21}+\left(\Gamma_{11}^{1}+\Gamma_{12}^{2}\right) \rho_{21}+\Gamma_{12}^{1} \rho_{11}\right] \\
& +\alpha_{1}^{2} \alpha_{2}\left[-\partial_{2} \rho_{21}-\partial_{1} \rho_{22}+\left(\Gamma_{12}^{1}+\Gamma_{22}^{2}\right) \rho_{21}\right. \\
& \left.+\Gamma_{22}^{1} \rho_{11}+3 \Gamma_{12}^{2} \rho_{22}+\left(\rho_{12}+\rho_{21}\right) \Gamma_{12}^{1}\right] \\
& +\alpha_{1} \alpha_{2}^{2}\left[-\partial_{2} \rho_{22}+2 \Gamma_{22}^{2} \rho_{22}+\left(\rho_{12}+\rho_{21}\right) \Gamma_{22}^{1}\right] \\
D= & \alpha_{1}^{3}\left[\partial_{1} \rho_{11}-2 \Gamma_{11}^{1} \rho_{11}-\left(\rho_{12}+\rho_{21}\right) \Gamma_{11}^{2}\right] \\
& +\alpha_{1}^{2} \alpha_{2}\left[\partial_{2} \rho_{11}+\partial_{1} \rho_{12}-3 \Gamma_{12}^{1} \rho_{11}-\Gamma_{11}^{2} \rho_{22}\right. \\
& \left.-\left(\Gamma_{11}^{1}+\Gamma_{12}^{2}\right) \rho_{12}-\left(\rho_{12}+\rho_{21}\right) \Gamma_{12}^{2}\right] \\
& +\alpha_{1} \alpha_{2}^{2}\left[\partial_{2} \rho_{12}-\Gamma_{22}^{1} \rho_{11}-\Gamma_{12}^{2} \rho_{22}-\left(\Gamma_{12}^{1}+\Gamma_{22}^{2}\right) \rho_{12}\right]
\end{aligned}
$$

Its characteristic polynomial is given by

$$
P_{\lambda}\left[S^{\nabla}(X)\right]=\lambda^{2}-\lambda(A+D)+(A D-B C) .
$$

Here, we investigate affine surfaces whose Ricci tensor is skew-symmetric.
Theorem 3. Let $\nabla$ be an torsion-free affine connection on a surface $\Sigma$. Then, the Ricci tensor of $\nabla$ is skew-symmetric and nonzero everywhere if and only if $(\Sigma, \nabla)$ is affine Szabó.

Proof. If the Ricci tensor of $\nabla$ is skew-symmetric, that is, $\rho_{11}=\rho_{22}=0$ and $\rho_{12}=-\rho_{21}$. Then, the Szabó operator is nilpotent.

Conversely, if $(\Sigma, \nabla)$ is affine Szabó, then the trace and determinant of (14) will be zero, which is possible only if $\rho_{11}=\rho_{22}=0$ and $\rho_{12}=-\rho_{21}$.

The investigation of affine connections with skewsymmetric Ricci tensor on surfaces has been extremely attractive and fruitful over the recent years. We refer to the paper [18] by Derdzinski for further details. Taking into account the simplified Wong's theorem ([19], Theorem 4.2) given in [18], we have the following.

Theorem 4. If every point of an affine surface $\Sigma$ has a neighborhood $U$ with coordinates $\left(u_{1}, u_{2}\right)$ in which the component functions of a torsion-free affine connection $\nabla$ are $\Gamma_{11}^{1}=-\partial_{1} \varphi, \Gamma_{22}^{2}=\partial_{2} \varphi$, for some function $\varphi, \Gamma_{j k}^{l}=0$, unless $j=k=l$, then $(\Sigma, \nabla)$ is affine Szabó.

Proof. It easy to show that the Ricci tensor of $\nabla$ is skewsymmetric.

A Lagrangian $L: U \longrightarrow \mathbb{R}$ in a manifold $\Sigma$ is a function on a nonempty open set $U \subset T \Sigma$. A Lagrangian $L: U \longrightarrow \mathbb{R}$ gives rise to equations of motion, which are the Euler-Lagrange equations, imposed on curves $t \longrightarrow y(t) \in \Sigma$ and the velocity $t \longrightarrow v(t) \in T \Sigma$ lies entirely in $U$. A fractional-linear function in a two-dimensional real vector space $\Pi$ is a rational function of the form $\alpha / \beta$, defined on a nonempty open subset of $\Pi / \operatorname{ker} \beta$, where $\alpha, \beta \in \Pi^{*}$ are linearly independent functionals. By using ([18], Theorem 11.1) and Theorem 3, we have.

Theorem 5. Let $\nabla$ be a torsion-free affine connection on a surface $\Sigma$. If every point in $T \Sigma \backslash \Sigma$ has a neighborhood $U$ with a fractional-linear Lagrangian $L: U \longrightarrow \mathbb{R}$ such that the solutions of the Euler-Lagrange equations for $L$ coincide with those geodesics of $\nabla$ which, lifted to $T \Sigma$, lie in $U$, then $(\Sigma, \nabla)$ is affine Szabó.

Definition 1 (see [19]). A tensor field $T$ is said to be recurrent if there exists a 1 -form $\alpha$ such that $\nabla T=\alpha \otimes T$, where $\nabla$ is an affine connection. In particular, an affine surface $(\Sigma, \nabla)$ is said to be recurrent if its Ricci tensor is recurrent.

Theorem 6. Let $(\Sigma, \nabla)$ be an affine Szabó surface. Then, $(\Sigma, \nabla)$ is recurrent if and only if, around each point, there exists a coordinate system $\left(U, u^{h}\right)$ with the nonzero components of $\nabla$ which are

$$
\begin{aligned}
& \Gamma_{11}^{1}=-\partial_{1} \varphi, \\
& \Gamma_{22}^{2}=\partial_{2} \varphi,
\end{aligned}
$$


for some scalar function $\varphi$ such that $\partial_{2} \partial_{1} \varphi \neq 0$. Moreover, $(\Sigma, \nabla)$ is not locally symmetric.

Proof. Consider the Ricci tensor $\rho=\rho_{a}+\rho_{s}$, where $\rho_{a}$ is the antisymmetric part of $\rho$ and $\rho_{s}$ is the symmetric part of $\rho$. Then, by using Theorem 3 , we can say that $(\Sigma, \nabla)$ is an affine Szabó if and only if the Ricci tensor of $\nabla$ is skew-symmetric and nonzero everywhere. Then, it follows from ([19], Theorem 4.2) that one of the three possibilities for a nonflat recurrent affine surface is the one in which, around each point, there exists a coordinate system $\left(U, u^{h}\right)$ with the nonzero components of $\nabla$ which are

$$
\begin{aligned}
& \Gamma_{11}^{1}=-\partial_{1} \varphi, \\
& \Gamma_{22}^{2}=\partial_{2} \varphi,
\end{aligned}
$$

for some scalar function $\varphi$ such that $\partial_{2} \partial_{1} \varphi \neq 0$. Now, it is easy to calculate that $\rho_{21}=-\rho_{12}=\partial_{2} \partial_{1} \varphi$, which is never zero. So, $(\Sigma, \nabla)$ is not locally symmetric.

By using the result of ([19], Theorem 2.2) and Theorem 3, we can say as follows.

Theorem 7. Let $(\Sigma, \nabla)$ be an affine Szabó recurrent surface. Then, the recurrence covector of a recurrence tensor is not locally a gradient.

\section{The Deformed Riemannian Extensions of an Affine Szabó Manifold}

A pseudo-Riemannian manifold $(M, g)$ is said to be Szabó if the Szabó operators $S(X)=\left(\nabla_{X} R\right)(\cdot, X) X$ have constant eigenvalues on the unit pseudosphere bundles $S^{ \pm}(T M)$. Any Szabó manifold is locally symmetric in the Riemannian [15] and the Lorentzian [20] setting, but the higher signature case supports examples with nilpotent Szabó operators (cf. [21] and the references therein). Now, we will prove the following result.

Theorem 8. Let $(M, \nabla)$ be a 2-dimensional smooth torsionfree affine manifold. Then, the following assertions are equivalent:

(1) $(M, \nabla)$ is an affine Szabó manifold

(2) The deformed Riemannian extension $\left(T^{*} M, g_{(\nabla, \phi)}\right)$ of $(M, \nabla)$ is a pseudo-Riemannian nilpotent Szabó manifold of neutral signature

Proof. Let $\Gamma_{i j}^{k}$ be the coefficients of the torsion-free affine connection $\nabla$ and $\phi_{i j}$ denote the local components of $\phi$. Then, the deformed Riemannian extension of the torsionfree affine connection $\nabla$ is the pseudo-Riemannian metric tensor on $T^{*} M$ of signature $(2,2)$ given by

$$
\begin{aligned}
g_{(\nabla, \phi)}= & \left(\phi_{11}\left(u_{1}, u_{2}\right)-2 u_{3} \Gamma_{11}^{1}-2 u_{4} \Gamma_{11}^{2}\right) \mathrm{d} u_{1} \otimes \mathrm{d} u_{1} \\
& +\left(\phi_{22}\left(u_{1}, u_{2}\right)-2 u_{3} \Gamma_{22}^{1}-2 u_{4} \Gamma_{22}^{2}\right) \mathrm{d} u_{2} \otimes \mathrm{d} u_{2} \\
& +\left(\phi_{12}\left(u_{1}, u_{2}\right)-2 u_{3} \Gamma_{12}^{1}-2 u_{4} \Gamma_{12}^{2}\right) \\
& \cdot\left(\mathrm{d} u_{1} \otimes \mathrm{d} u_{2}+\mathrm{d} u_{1} \otimes \mathrm{d} u_{2}\right) \\
& +\left(\mathrm{d} u_{1} \otimes \mathrm{d} u_{3}+\mathrm{d} u_{3} \otimes \mathrm{d} u_{1}+\mathrm{d} u_{2} \otimes \mathrm{d} u_{4}+\mathrm{d} u_{4} \otimes \mathrm{d} u_{2}\right) .
\end{aligned}
$$

A straightforward calculation shows that the nonzero Christoffel symbols $\widetilde{\Gamma}_{\alpha \beta}^{\gamma}$ of the Levi-Civita connection are given as follows:

$$
\begin{aligned}
& \widetilde{\Gamma}_{i j}^{k} \Gamma_{i j}^{k} ; \widetilde{\Gamma}_{i^{\prime} j}^{k^{\prime}}=-\Gamma_{j k}^{i} ; \widetilde{\Gamma}_{i j^{\prime}}^{k^{\prime}}=-\Gamma_{i j}^{j}, \\
& \widetilde{\Gamma}_{i j}^{k^{\prime}} \sum_{r=1}^{2}\left(\partial_{k} \Gamma_{i j}^{r}-\partial_{i} \Gamma_{j k}^{r}-\partial_{j} \Gamma_{i k}^{r}+2 \sum_{l=1}^{2} \Gamma_{k l}^{r} \Gamma_{i j}^{l}\right) \phi_{k l} \Gamma_{i j}^{l}, \\
& \quad+\frac{1}{2}\left(\partial_{i} \phi_{j k}+\partial_{j} \phi_{i k}-\partial_{k} \phi_{i j}\right)-\sum_{l=1}^{2}
\end{aligned}
$$

where $(i, j, k, l, r=1,2) \quad$ and $\quad\left(i^{\prime}=i+2, j^{\prime}=j+2, k^{\prime}\right.$ $\left.=k+2, r^{\prime}=r+2\right)$. The nonzero components of the curvature tensor of $\left(T^{*} M, g_{(\nabla, \phi)}\right)$ up to the usual symmetries are given as follows (we omit $\widetilde{R}_{k j i}^{h^{\prime}}$, as it plays no role in our considerations):

$$
\begin{aligned}
\widetilde{R}_{k j i}^{h} & =R_{k j i}^{h}, \\
\widetilde{R}_{k j i}^{h^{\prime}}, \widetilde{R}_{k j i}^{h^{\prime}}= & -R_{k j h}^{i}, \\
\widetilde{R}_{k^{\prime} j i}^{h^{\prime}} & =R_{h i j}^{k},
\end{aligned}
$$

where $R_{k j i}^{h}$ are the components of the curvature tensor of $(M, \nabla)$. For more details, see [14].

Let $\widetilde{X}=\alpha_{i} \partial_{i}+\alpha_{i^{\prime}} \partial_{i^{\prime}}$ be a vector field on $T^{*} M$. Then, the matrix of the Szabó operator $\widetilde{S}(\widetilde{X})$ with respect to the basis $\left\{\partial_{i}, \partial_{i^{\prime}}\right\}$ is of the form:

$$
\widetilde{S}(\widetilde{X})=\left(\begin{array}{cc}
S^{\nabla}(X) & 0 \\
* & { }^{t} S^{\nabla}
\end{array}(X)\right),
$$

where $S^{\nabla}(X)$ is the matrix of the affine Szabó operator on $M$ relative to the basis $\left\{\partial_{i}\right\}$. Note that the characteristic polynomial $P_{\lambda}[\widetilde{S}(\tilde{X})]$ of $\tilde{S}(\tilde{X})$ and $P_{\lambda}\left[S^{\nabla}(X)\right]$ of $S^{\nabla}(X)$ are related by

$$
P_{\lambda}[\widetilde{S}(\widetilde{X})]=P_{\lambda}\left[S^{\nabla}(X)\right] \cdot P_{\lambda}\left[{ }^{t} S^{\nabla}(X)\right] .
$$

Now, if the affine manifold $(M, \nabla)$ is assumed to be affine Szabó, then $S^{\nabla}(X)$ has zero eigenvalues for each vector field $X$ on $M$. Therefore, it follows from (23) that the eigenvalues of $\widetilde{S}(\widetilde{X})$ vanish for every vector field $\widetilde{X}$ on $T^{*} M$. Thus, $\left(T^{*} M, g_{\nabla}\right)$ is pseudo-Riemannian Szabó manifold.

Conversely, assume that $\left(T^{*} M, g_{\nabla}\right)$ is a pseudo-Riemannian Szabó manifold. If $X=\alpha_{i} \partial_{i}$ is an arbitrary vector 
field on $M$, then $\widetilde{X}=\alpha_{i} \partial_{i}+\left(1 / 2 \alpha_{i}\right) \partial_{i^{\prime}}$ is an unit vector field at every point of the zero section on $T^{*} M$. Then, from (23), we see that the characteristic polynomial $P_{\lambda}[\widetilde{S}(\widetilde{X})]$ of $\widetilde{S}(\widetilde{X})$ is the square of the characteristic polynomial $P_{\lambda}\left[S^{\nabla}(X)\right]$ of $S^{\nabla}(X)$. Since for every unit vector field $\widetilde{X}$ on $T^{*} M$, the characteristic polynomial $P_{\lambda}[\widetilde{S}(\widetilde{X})]$ should be the same, and it follows that, for every vector field $X$ on $M$, the characteristic polynomial $P_{\lambda}\left[S^{\nabla}(X)\right]$ is the same. Hence, $(M, \nabla)$ is affine Szabó.

For an example, we have the following.

Theorem 9 (see [6]). Let $M=\mathbb{R}^{2}$ and $\nabla$ be the torsion-free connection defined by $\nabla_{\partial_{1}} \partial_{1}=f_{1}\left(u_{1}\right) \partial_{2}$ and $\nabla_{\partial_{1}} \partial_{2}=f_{2}\left(u_{1}\right) \partial_{2}$. Assume that $f_{1}$ and $f_{2}$ satisfies $\partial_{1} b=0$ and $\partial_{2} b=0$, where $b=\partial_{1} f_{2}+f_{2}^{2}$. Then, the pseudo-Riemannian metric $g_{(\nabla, \phi)}$ on the cotangent bundle $T^{*} M$ of neutral signature $(2,2)$ defined by setting such that

$$
\begin{aligned}
g_{(\nabla, \phi)}= & \left(\phi_{11}-2 u_{4} f_{1}\right) d u_{1} \otimes d u_{1}+\phi_{22} d u_{2} \otimes d u_{2} \\
& +\left(\phi_{12}-2 u_{4} f_{2}\right)\left(d u_{1} \otimes d u_{2}+d u_{1} \otimes d u_{2}\right) \\
& +\left(d u_{1} \otimes d u_{3}+d u_{3} \otimes d u_{1}+d u_{2} \otimes d u_{4}+d u_{4} \otimes d u_{2}\right),
\end{aligned}
$$

is Szabó for any symmetric $(0,2)$-tensor field $\phi$.

\section{Data Availability}

No data were used to support this study.

\section{Conflicts of Interest}

The authors declare that they have no conflicts of interest.

\section{References}

[1] E. M. Patterson and A. G. Walker, "Riemann extensions," The Quarterly Journal of Mathematics, vol. 3, no. 1, pp. 19-28, 1952.

[2] Z. Afifi, "Riemann extensions of affine connected spaces," The Quarterly Journal of Mathematics, vol. 5, no. 1, pp. 312-320, 1954.

[3] E. García-Río, D. N. Kupeli, M. E. Vázquez-Abal, and R. Vázquez-Lorenzo, "Affine Osserman connections and their Riemann extensions," Differential Geometry and Its Applications, vol. 11, no. 2, pp. 145-153, 1999.

[4] A. S. Diallo, "The Riemann extension of an affine Osserman connection on 3-dimensional manifolds," Global Journal of Advanced Research on Clasical and Modern Geometry, vol. 2, no. 2, pp. 69-75, 2013.

[5] E. García-Río, P. Gilkey, S. Nikcević, and R. VázquezLorenzo, "Applications of affine and Weyl geometry," in Synthesis Lectures on Mathematics and StatisticsMorgan \& Claypool Publishers, Williston, VT, USA, 2013.

[6] A. S. Diallo and P. Gupta, "On a deformed Riemannian extension of affine Szabó connections," Balkan Journal of Geometry and its Applications, vol. 25, no. 2, pp. 30-38, 2020.

[7] O. Kowalski and M. Sekizawa, "On natural Riemann extensions," Publicationes Mathematicae Debrecen, vol. 78, no. 3-4, pp. 709-721, 2011.
[8] O. Kowalski and M. Sekizawa, "The Riemann extensions with cyclic parallel Ricci tensor," Mathematische Nachrichten, vol. 287, no. 8-9, pp. 955-961, 2014.

[9] L. Vanhecke and T. J. Willmore, "Riemann extensions of D’Atri spaces," Tensor (N.S.), vol. 38, pp. 154-158, 1982.

[10] A. Bonome, R. Castro, E. García-Río, L. Hervella, and R. Vázquez-Lorenzo, "Nonsymmetric Osserman indefinite Kähler manifolds," Proceedings of the American Mathematical Society, vol. 126, no. 9, pp. 2763-2769, 1998.

[11] M. Brozos-Vázquez, E. García-Río, P. Gilkey, S. Nikcević, and R. Vézquez-Lorenzo, The Geometry of Walker Manifolds, Synthesis Lectures on Mathematics and Statistics 5, Morgan \& Claypool Publ., Williston, VT, USA, 2009.

[12] A. S. Diallo, M. Hassirou, and I. Katambe, "The twisted Riemannian extension of an affine Osserman connection on 2-dimensional manifolds," Mathematical Sciences Research Journal, vol. 17, no. 8, pp. 201-207, 2013.

[13] K. Yano and S. Ishihara, "Tangent and cotangent bundles: differential geometry," in Pure and Applied MathematicsMarcel Dekker, New York, NY, USA, 1973.

[14] E. Calviño-Louzao, E. García-Río, and R. Vázquez-Lorenzo, "Riemann extensions of torsion-free connections with degenerate Ricci tensor," Canadian Journal of Mathematics, vol. 62, no. 5, pp. 1037-1057, 2010.

[15] Z. I. Szabó, "A short topological proof for the symmetry of 2 point homogeneous spaces," Inventiones Mathematicae, vol. 106, pp. 61-64, 1991.

[16] A. S. Diallo, S. Longwap, and F. Massamba, "On three dimensional affine Szabó manifolds," Balkan Journal of Geometry and Its Applications, vol. 22, no. 2, pp. 21-36, 2017.

[17] A. S. Diallo and F. Massamba, "Affine Szab ó connections on smooth manifolds," Revista de la Unión Matemática Argentina, vol. 58, no. 1, pp. 37-52, 2017.

[18] A. Derdzinski, "Connections with skew-symmetric Ricci tensor on surfaces," Results in Mathematics, vol. 52, no. 3-4, pp. 223-245, 2008.

[19] Y.-C. Wong, "Two dimensional linear connexions with zero torsion and recurrent curvature," Monatshefte für Mathematik, vol. 68, no. 2, pp. 175-184, 1964.

[20] P. Gilkey and I. Stavrov, "Curvature tensors whose Jacobi or Szabó operator is nilpotent on null vectors," Bulletin of the London Mathematical Society, vol. 34, no. 06, pp. 650-658, 2002.

[21] P. B. Gilkey, R. Ivanova, and T. Zhang, "Szabó osserman IP pseudo-riemannian manifolds," Publicationes Mathematicae Debrecen, vol. 62, no. 3-4, pp. 387-401, 2003. 